\title{
Possible Origins of the Complex Topographic Organization of Motor Cortex: Reduction of a Multidimensional Space onto a Two-Dimensional Array
}

\author{
Tyson N. Aflalo and Michael S. A. Graziano \\ Department of Psychology, Princeton University, Princeton, New Jersey 08544-1010
}

\begin{abstract}
We propose that some of the features of the topographic organization in motor cortex emerge from a competition among several conflicting mapping requisites. These competing requisites include a somatotopic map of the body, a map of hand location in space, and a partitioning of cortex into regions that emphasize different complex, ethologically relevant movements. No one type of map fully explains the topography; instead, all three influences (and perhaps others untested here) interact to form the topography. A standard algorithm (Kohonen network) was used to generate an artificial motor cortex array that optimized local continuity for these conflicting mapping requisites. The resultant hybrid map contained many features seen in actual motor cortex, including the following: a rough, overlapping somatotopy; a posterior strip in which simpler movements were represented and more somatotopic segregation was observed, and an anterior strip in which more complex, multisegmental movements were represented and the somatotopy was less segregated; a clustering of different complex, multisegmental movements into specific subregions of cortex that resembled the arrangement of subregions found in the monkey; three hand representations arranged on the cortex in a manner similar to the primary motor, dorsal premotor, and ventral premotor hand areas in the monkey; and maps of hand location that approximately matched the maps observed in the monkey.
\end{abstract}

Key words: primary motor; premotor; reaching; grasping; avoidance; motor learning

\section{Introduction}

Many different variants of functional topography have been proposed for the lateral motor strip in the primate precentral gyrus. Some of these variants are shown in Figure 1. These proposed organizations include versions of a somatotopic map (see Fig. $1 A-C$ ) (Foerster, 1936; Woolsey et al., 1952; Kwan et al., 1978; Park et al., 2001) and divisions of the precentral gyrus into primary motor cortex and several sectors of caudal and rostral premotor cortex (see Fig. 1D-F) (Campbell, 1905; Matelli et al., 1985; Preuss et al., 1996; Dum and Strick, 2001, 2005). Two additional types of organization were suggested by recent experiments using electrical stimulation. We mapped the motor cortex in monkeys using long-train electrical stimulation to evoke complex, multijoint movements (Graziano et al., 2002, 2003, 2004, 2005; Cooke and Graziano, 2004a). As expected, we obtained a rough, overlapping somatotopic organization consistent with previous studies, with the feet in a medial location and the mouth in a lateral location. In addition, within the arm and hand representation, we obtained a rough map of the location in space to which the hand moved on stimulation (see Fig. 1G,H) (Graziano

Received Feb. 20, 2006; revised April 26, 2006; accepted April 26, 2006

This work was supported by National Institutes of Health Grant NS-046407 and by Burroughs Wellcome Grant 992817. We thank T. Mole and J. McCain.

Correspondence should be addressed to Michael S. A. Graziano, Department of Psychology, Princeton University, Princeton, NJ 08544-1010. E-mail: graziano@princeton.edu.

DOI:10.1523/JNEUROSCI.0768-06.2006

Copyright $\odot 2006$ Society for Neuroscience $\quad 0270-6474 / 06 / 266288-10 \$ 15.00 / 0$ et al., 2002, 2005). This hand location map encompassed the traditional primary motor cortex and the caudal parts of premotor cortex. We also obtained a partitioning of motor and premotor cortex into zones that emphasized different complex, ethologically relevant movements (see Fig. 1I) (Graziano et al., 2002, 2005). These additional topographies were unexpected but may help to explain the noise and overlapping within the somatotopic progression. We hypothesized that the simultaneous presence of several noisy and intermeshed types of topographic organization might be the result of a competition among conflicting mapping requisites (Graziano et al., 2005).

Kohonen $(1984,2001)$ suggested that cortical maps may selforganize to optimize nearest-neighbor relationships. In this view, neurons that process similar information are located near each other, minimizing wiring length. Self-organization can lead to fractured or seemingly disordered maps in certain cases because of the fact that the cortex is two-dimensional, yet the relevant parameter space may be of higher dimensionality. For example, the principle of self-organizing maps was used to explain the complex organization of primary visual cortex (Durbin and Mitchison, 1990), in which line orientation and ocular dominance interact to produce a pattern of cortical pinwheels (Obermayer and Blasdel, 1993). Likewise, some of the apparent disorder in the tonotopic map in primary auditory cortex has been attributed to a self-organization that is under the influence of many dimensions reduced onto a two-dimensional sheet (Schreiner, 1995). 
A

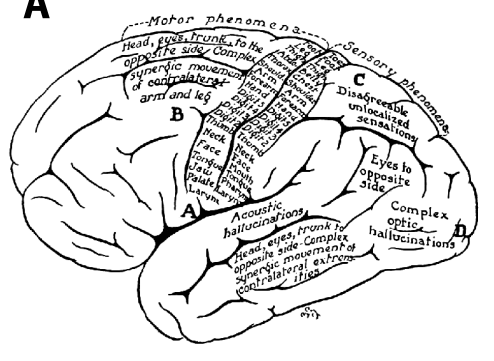

B

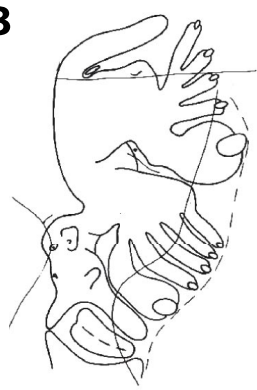

$\mathbf{E}$

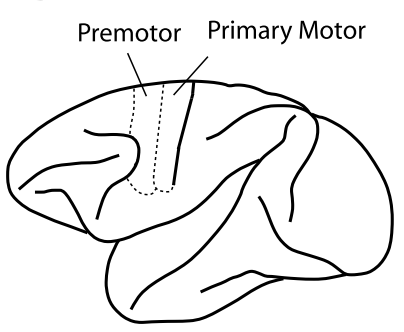

\section{G}

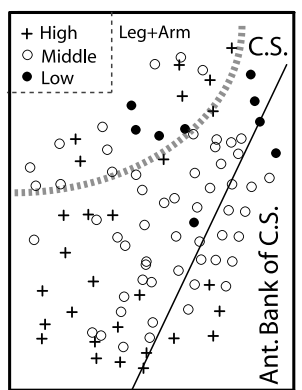

H

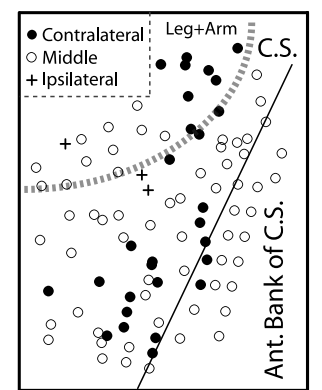

I

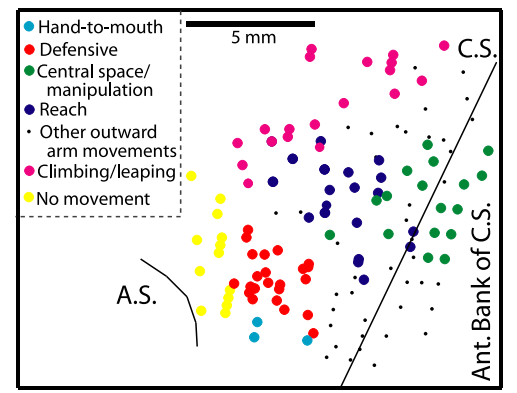

Figure 1. Eight different possible organizations to the lateral motor cortex in the monkey brain. $A$, A sequence of body parts arranged vertically with the feet in a medial location and the mouth in a lateral location (Fritsch and Hitzig, 1870; Foerster, 1936; Fulton, 1938). $\boldsymbol{B}$, A subsequent refinement of the map including a horizontal component to the organization, in which the axial musculature is represented anteriorly and the distal musculature posteriorly (Woolsey et al., 1952). C, Another proposed organization in which a posterior hand representation is partly surrounded by an arm representation (Kwan et al., 1978; Park et al., 2001). D, Division of the precentral gyrus into two strips: primary motor cortex that tended to represent individual body parts and the more complex premotor cortex (Campbell, 1905). E, Partitioning of premotor cortex into a dorsal and ventral sector, each sector subdivided into a caudal and a rostral part (Matelli et al., 1985; Preuss et al., 1996). $\boldsymbol{F}$, Three regions in the lateral motor strip that project directly to the hand portion of the spinal cord: the primary motor hand area, the ventral premotor hand area, and the dorsal premotor hand area (Dum and Strick, 2002, 2005). G, Rough map of the height of the hand as evoked by electrical stimulation. Data from one monkey (Graziano et al., 2005). Upper hand locations are in a ventral location, and lower hand locations are in a dorsal location. Within the arm-leg region of the somatotopy, the mapping of the hand height breaks down. $\boldsymbol{H}$, Data from the same monkey as in $\mathbf{G}$ but now showing the lateral position of hand evoked by electrical stimulation. $\mathbf{I}$, Cortical patches that, when stimulated, result in different ethologically relevant movements. The details differ among monkeys, but the overall pattern is consistent. Data from one monkey (Graziano et al., 2005). A.S., Arcuate sulcus; C.S., central sulcus; F4/F5, fields 4 and 5 of motor cortex; M1, primary motor cortex; PMd, dorsal promotor area; PMv, ventral premotor area; PMDc, dorsal premotor cortex, caudal division; PMDr, dorsal premotor cortex, rostral division; SMA, supplementary motor area.

In the present study, we modeled motor cortex topography using a Kohonen network. We gave the map an initial somatotopic organization. We then allowed the network to reorganize under the influence of two additional mapping requisites: hand location in space, and ethologically relevant action categories that combine more than one body part. We hypothesized that the constraint of optimizing nearest-neighbor relationships in this multidimensional space would force the map to express many of the specific, complex features found in the monkey motor cortex.
C

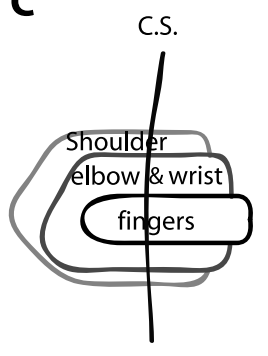

$\mathbf{F}$

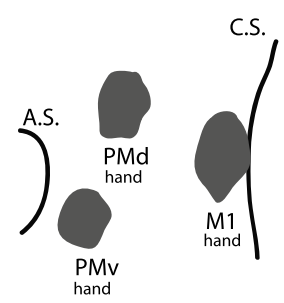

\section{Materials and Methods}

The Kohenen network is a standard tool for solving the problem of dimensionality reduction or the problem of representing a multidimensional space on a lower dimensional space such that neighbor relationships are optimized. For the present purpose, the self-organizing Kohonen network is not meant to model the specific neuronal interactions or learning algorithms of the brain. Rather, the network is merely an analytic tool that optimizes topographic continuity. No assumptions are made here about the learning algorithms that might be implemented in the brain to produce smoothly varying topographic maps.

The final product of a traditional selforganizing map algorithm is an array of nodes that provides a structured representation of the input data. Each node represents a subset of the input data, and nodes that are topographically near each other in the array tend to represent similar input data. This topographic feature is the natural result of the learning algorithm: when an input is presented to the network, the node that best matches that input, and the topographically surrounding nodes, are updated to more closely represent that input. This local smoothing effect leads to a global structuring of the data.

In our study, the array of nodes was a $36 \times 20$ two-dimensional grid that formed the artificial motor cortex. Each node represented a movement. This movement representation was expressed in the form of an 18-dimensional vector, called a codebook vector. Each vector specified the body parts that were being moved (10 dimensions, one for each body part), the position that the hand reached in Cartesian space (three dimensions), and the ethological category to which the movement belonged (five dimensions corresponding to five categories). These aspects of the codebook vector are explained in the sections below.

Somatotopy. Figures 1 and $2 A$ show Woolsey's representation of the somatotopic organization of motor cortex in the monkey (Woolsey et al., 1952). This somatotopy is one of the most complete in the literature because it covers most of the precentral gyrus and shows a horizontal (trunk to hand) as well as vertical (head to toe) organization. This somatotopy is of course only approximate and leaves out details of overlapping and repeated representations commonly reported in the monkey. We took this simple, global somatotopy as a starting point for the self-organizing map. The rationale for beginning with a simple somatotopy was the developmental studies of Martin et al. (2005) showing that motor cortex may indeed begin with a prewired somatotopy that is then altered and blurred through learning. As described in Results, during learning, this initial simple somatotopy developed into a more complex one with overlapping and repeated representations.

The overlay in Figure $2 \mathrm{~A}$ depicts 10 discrete somatotopic subregions corresponding to the tongue, lips, jaw, upper face, neck, torso, arm, hand, leg, and toe, approximately matching the underlying map. A schematized, blocked version of the Woolsey map was used for simplicity because the exact borders of the initial somatotopic components turned out to have little effect on the final state of the network. These somato- 
A

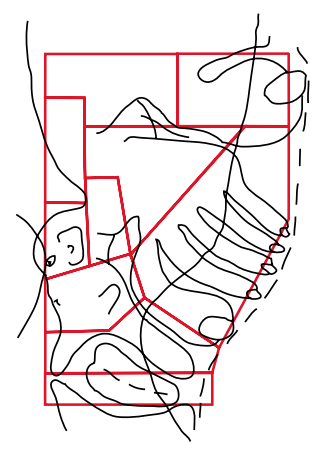

C

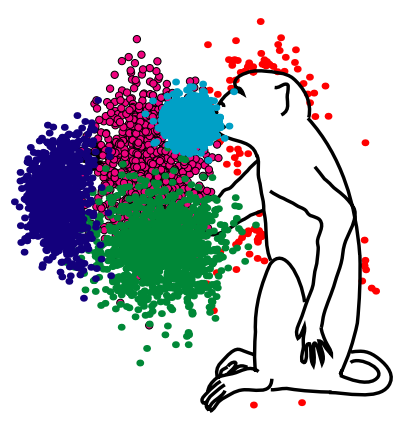

D

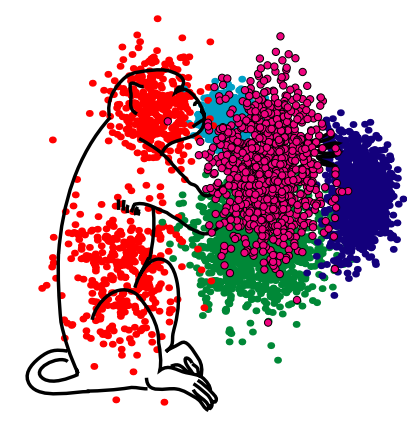

Figure 2. Some movement definitions used in the motor cortex model. $\boldsymbol{A}$, Woolsey's map of the somatotopic organization in monkey motor cortex (Woolsey et al., 1952), overlaid with blocked regions showing the schematized somatotopy used in the present motor cortex model. $\boldsymbol{B}-\boldsymbol{D}$, Three views of a schematized monkey showing the hand locations defined for different ethological categories of movement. Light blue, Hand-to-mouth; dark blue, reach; red, defense; green, central space/manipulation; pink with black border, climbing.

topic regions covered a rectangular area with a missing wedge in the posterior lateral corner. Therefore, our node array had this same shape of a rectangular area with a missing wedge in the posterior lateral corner. To operationalize the somatotopy in our node array, a 10-element codebook vector was created with each element corresponding to a particular body part. This vector took the form of [tongue, lips, jaw, upper face, neck, torso, arm, hand, toe, leg]. As an initial state, before any learning, each node in the array was assigned a codebook vector in which body parts took a value of either 0 , indicating that the node did not represent that particular body part, or 1 , indicating that the node did represent the particular body part. In this way, the array was initialized to match the Woolsey map. For example, nodes in the hand area were initially characterized as $[0,0,0,0,0,0,0,1,0,0]$. During learning, these initial values could change in a continuous manner. In this way, the initial, cleanly segregated somatotopy could be altered and nodes could come to partially represent any combination of body parts.

Hand position. Hand position was represented by adding three dimensions ( $x, y$, and $z$ positions) to the 10-dimensional somatotopic description. All hand positions were encoded in centimeters relative to the mouth, with positive $x$ as the vertical height in centimeters above the mouth, positive $y$ as the horizontal distance in the contralateral direction away from the mouth, and positive $z$ as the distance forward of the mouth along the line of sight. For example, a node representing hand position in lower, central space might be characterized as $[-20,0,0]$. Hand position was only included for those nodes at which the arm component of the somatotopy was non- 0 . If the arm component had a value of 0 , then the values for hand position were left empty and did not actively contribute to the formation of the topographic map. Hand positions were initialized in the node array as small random numbers uniformly distributed be- tween -0.1 and 0.1 . Thus, initially, the array contained no topographic map of hand position.

Ethological category. Finally, movements were categorized according to an ethological description of the movement. These ethological categories were based on our previous finding of a set of discrete movement categories evoked from specific sites in motor cortex. Each category of movement included a range of different movements all similar in that they served a common behavioral function. To represent these categories, we used a five-dimensional vector, each dimension corresponding to a particular category of behavior. For example, $[1,0,0,0,0]$ indicated a movement that belonged entirely to the first ethological category but not to any of the other categories. Ethological categories were initialized in the array as small random numbers uniformly distributed between -0.1 and 0.1 . Thus, initially, the array contained no systematic representation of ethological categories. The five categories are described in the following sections.

Hand-to-mouth movements. Hand-to-mouth movements are common in the natural behavior of monkeys (Graziano et al., 2003) and were also obtained on electrical stimulation of certain regions of the motor cortex (Graziano et al., 2002, 2005). These movements include a closure of the hand into a grip posture, a movement of the hand toward the mouth, a movement of the mouth, and a rotation of the head to bring the mouth in alignment with the hand. In our self-organizing network, this type of movement was defined as combining the tongue, lips, jaw, neck, arm, and hand. The distribution of hand positions associated with handto-mouth movements was defined as having a mean position of $[0,0,3]$, with SDs of $[1,1,1]$. This distribution approximates the distribution of hand-to-mouth movements obtained on stimulation of the monkey cortex and is shown by the light blue dots in Figure $2 B-D$. A node in the motor cortex array that represents specifically and only a hand-to-mouth movement might have a codebook vector of $[(1,1,1,0,1,0,1,1,0,0)$ $(0.5,0,1)(1,0,0,0,0)]$ in which the first set of parentheses shows the somatotopic components, the second set shows the hand location, and the third set shows the ethological category. Including an explicit representation of ethological category in the codebook array allows movements that are different from each other to nonetheless belong to the same ethological category; as a result, during reorganization, these movements tend to become represented near each other.

Defensive movements. Three different but related types of movement were included in the ethological category of defensive movement. The first type was a facial defensive movement. This movement was studied in monkeys exposed to an air puff on the face (Cooke and Graziano, 2003) and was also observed on chemical or electrical stimulation of specific parts of motor cortex (Graziano et al., 2002, 2005; Cooke and Graziano, 2004a,b). The movement included a blink, a squinting of the musculature around the eyes, a lifting of the upper lip and sometimes opening of the jaw, a folding of the ear against the side of the head, and a retraction of the head. In our self-organizing network, therefore, this type of defensive movement was defined as combining the lips, jaw, upper face, and neck. No arm movement or hand position was included. A node in the array that represents this kind of defensive movement might have a codebook vector of $[(0,1,1,1,1,0,0,0,0,0)(-,-,-)(0,1,0,0,0)]$, in which the three dimensions of hand position in space are left blank because the arm is not involved (arm component, 0 ).

The second type of defensive movement included a facial and arm movement. This movement was also observed in monkeys exposed to an air puff to the face (Cooke and Graziano, 2003) and was observed on chemical or electrical stimulation of specific parts of motor cortex (Graziano et al., 2002, 2005; Cooke and Graziano, 2004a,b). The movement included all of the components of a facial defensive movement but also included a shrugging of the shoulder and a lifting of the hand to a region of space near the face as if to block an impending impact. In our selforganizing network, therefore, this type of defensive movement was defined as combining the lips, jaw, upper face, neck, torso, and arm. It was also defined as involving a final hand position in upper space near the side of the face. A node in the array that represents this kind of defensive movement might have a codebook vector of $[(0,1,1,1,1,1,1,0,0,0)$ $(2,8,0)(0,1,0,0,0)]$.

The third type of defensive movement was an arm retraction. This 
movement was typical of monkeys exposed to air puff on the arm or hand (Cooke and Graziano, 2003) and was also observed on chemical or electrical stimulation of specific regions in cortex (Graziano et al., 2002, 2005; Cooke and Graziano, 2004a,b). This movement involved a rapid withdrawal of the hand to a region at the side or back of the torso, accompanied by a twisting motion of the torso. In our self-organizing network, therefore, this type of defensive movement was defined as combining the torso and arm and involving hand positions in the lower space near the side or back of the torso. The hand positions related to defensive movement, as defined for the purposes of this model, are shown in Figure $2 B-D$ (red dots). A node in the array that represents this kind of defensive movement might have a codebook vector of $[(0,0,0,0,0,1,1,0,0,0)$ $(-20,8,0)(0,1,0,0,0)]$.

Central space manipulation. In monkeys, manipulation of objects such as food or toys typically occurs in a large ball of central space in front of the chest (Graziano et al., 2003). Stimulation of specific regions in the motor cortex of monkeys evoked movements of the fingers and wrist that resembled manipulation and also evoked accompanying movements of the arm that brought the hand into central space (Graziano et al., 2002, 2005). In our self-organizing network, this category of movement was defined as combining the hand and arm to varying proportions. Hand positions were given a mean position of $[-12,0,6]$, with SDs of $[3,3,3]$ (Fig. $2 B-D$, green dots). A node that represents a manipulation movement might have a codebook vector of $[(0,0,0,0,0,0,1,1,0,0)$ $(-10,0,10)(0,0,1,0,0)]$.

Reaching. Another salient type of movement observed on stimulation of motor cortex was a reaching-like movement in which the hand shaped as if to grasp an object, the arm projected outward, and the shoulder and torso rotated to contribute to the reach (Graziano et al., 2005). In our self-organizing network, this category of movement was defined as combining the hand, arm, and torso. The mean hand position was defined as $[-7,0,16]$. The outer boundary of this distribution of hand positions was made to follow a curve that roughly matched the expected boundary of a monkey's reach (outer diameter of curve, $30 \mathrm{~cm}$ ) (Fig. $2 B-D$, dark blue dots). A node in the array that represents a reach-to-grasp movement might have a codebook vector of $[(0,0,0,0,0,1,1,1,0,0)$ $(-6,0.2,17)(0,0,0,1,0)]$.

Climbing. A final type of complex movement observed on stimulation of motor cortex was a climbing-like movement that included all four limbs and the torso (Graziano et al., 2005). For example, during stimulation of a climbing-like site, the monkey might raise both arms, placing the hands into upper lateral space, push down on the contralateral leg as though to push off the ground, and raise the ipsilateral leg. In our selforganizing network, this category of movement was defined as combining the arm, leg, and torso. Hand position was given a mean of $[-5,7,7]$, with SDs of $[4,2,2]$ (Fig. $2 B-D$, pink dots with black border). A node in the array that represents a climbing movement might have a codebook vector of $[(0,0,0,0,0,1,1,0,1,0)(-4,8,7)(0,0,0,0,1)]$.

The definitions of movements given above incorporate specific details taken from the monkey data. For example, for purposes of the model, the central-space/manipulation movements are defined in a specific manner and given specific characteristics borrowed from the movements evoked from monkey cortex and the movements observed in the spontaneous behavior of monkeys. One possible concern is that the details of these movements might not be perfectly accurate. Should the central-space/ manipulation movements be centered exactly at a mean position of $[-12,0,6]$, with SDs of $[3,3,3]$ ? Should reaching movements extend to a furthest limit of $30 \mathrm{~cm}$ ? Would altering these details of the model slightly cause a substantial change in the results? As described in Results, the model turned out to be highly robust despite changes in these details of the movement definitions. As long as hand-to-mouth movements are roughly near the mouth, and a high percentage of manipulation takes place roughly in front of the chest, and the other movement categories are also roughly in the expected range, then the result is essentially the same.

Input vectors. As described above, each node in the network was assigned an initial codebook vector. The codebook vector indicated the movement or combination of movements currently represented by that location in cortex. We then defined a set of input vectors. The input vectors were the movement set on which the network was trained. Each input vector represented a specific movement and took the same form as a codebook vector. For example, an input vector of $[(1,1,1,0,1,0,1,1,0,0)(0.5,0,1)(1,0,0,0,0)]$ would represent a hand-tomouth movement.

The network was trained on 12,800 different input vectors. These input vectors included examples drawn in equal proportion from the ethological categories. To form a more complete, realistic movement set, the input vectors also included the following: movements of the foot and leg; movements of the mouth that combined the lips, jaw, and tongue (to mimic chewing); and movements of the arm to a sampling of locations in space. Monkeys spend a high proportion of time with the hand placed in three typical positions: the space just in front of the chest (such as for manipulation), the space just in front of the mouth (such as for manipulating food at the mouth), and lower space (such as for bracing the weight of the body during sitting) (Graziano et al., 2003). We constructed the input array to contain an equal number of vectors in which hand location was in central space, in front of the mouth, and in lower space, to include the normal range of a monkey's movements.

The exact proportions of these different types of movements turned out not to be critical. As described in Results, the final map was similar even when the proportion of movements was altered from equal proportions of different movement types to proportions that more closely approximated the normal repertoire of a monkey.

Learning rules. For details of the self-organizing learning algorithm and its implementation, see Kohonen (2001). Here we describe only those aspects of the technique for which there was more than one possible way to apply the method, requiring us to choose the approach that suited our particular application.

Before the learning algorithm was applied, each dimension of the input array was normalized such that values varied between -1 and 1 . In this manner, no dimension of the array could dominate the learning process (e.g., ensuring that the height of the hand had no more influence over map topology than any other dimension in the input vector).

The learning algorithm used was the batch computation variant of the self-organizing map. In the batch computation algorithm, the entire set of input data are used during each update step of the nodes of the network. The network is updated over multiple steps (in our case, 2000 steps). The alternative approach, an update rule in which the different input vectors are presented separately and in sequence, can produce results that are biased toward the particular inputs that happen to be presented early. The batch computation approach does not suffer from this problem of bias toward early inputs.

A defining feature of the self-organizing map is that, as each node of the map updates, it also updates its surrounding nodes. The extent to which nodes influence each other is given by this "neighborhood" function of which several forms can be applied. Following one standard method, we used the Gaussian neighborhood function that takes the following form:

$$
h_{c, i}=\exp \left(\frac{-d^{2}(c, i)}{2 \sigma^{2}(t)}\right)
$$

where $c$ and $i$ specify two nodes, $d(c, i)$ specifies the physical distance between the two nodes in the array, and $\sigma(t)$ specifies the timedependent SD of the Gaussian. The time dependence on $\sigma$ allows for a large initial neighborhood for the purposes of a global reordering of the network. The neighborhood then shrinks with time to allow for convergence on a stable solution. In our case, following standard conventions, the initial value of $\sigma$ was set to half the network size and fell off linearly for each iteration of the algorithm to a final value of 1 .

Often, various weighting schemes are used with self-organizing maps. Certain dimensions are weighted more than others, effectively making them dominant, when it is thought that they should have more effect on network topography. We used a standard weighting scheme in our network in which the somatotopic components of the codebook vector were updated at a rate $30 \%$ slower than the other components, providing for a more stable somatotopic organization. This weighting avoided situations in which the network, during reorganization, developed nonphysiologi- 
A

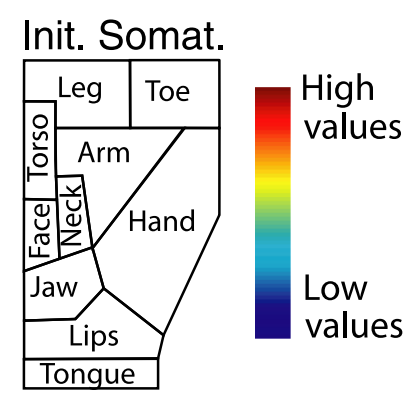

H
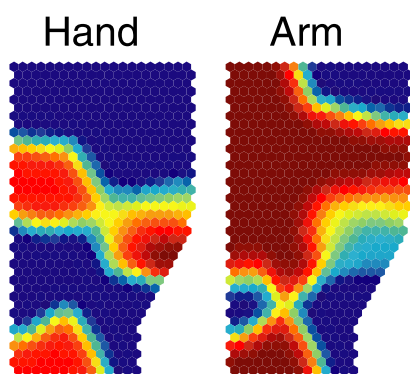

B

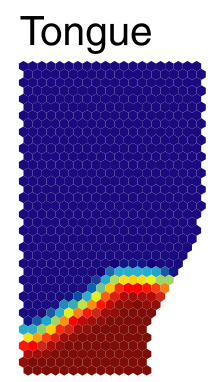

J
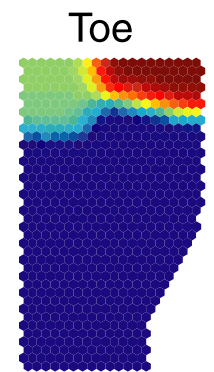

C

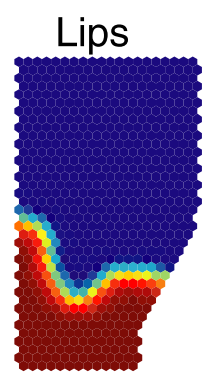

K
D

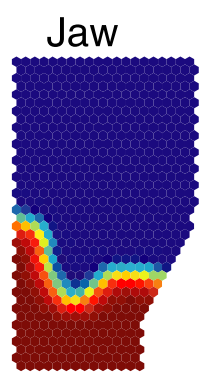

$\mathbf{L}$ Motion $\mathbf{M}$

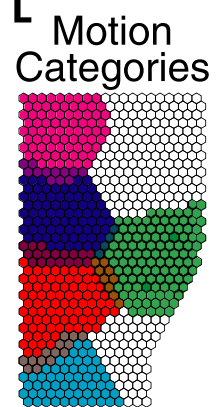

E
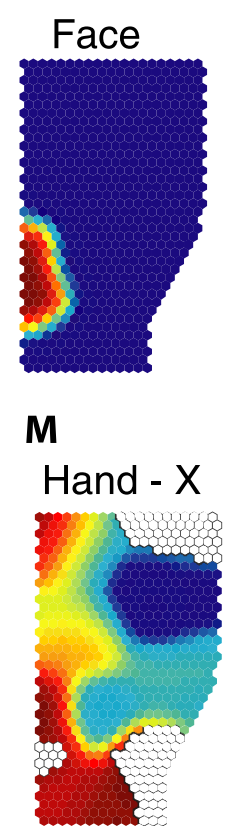

$\mathbf{F}$

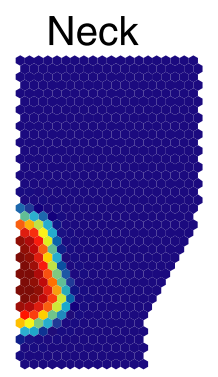

$\mathbf{N}$

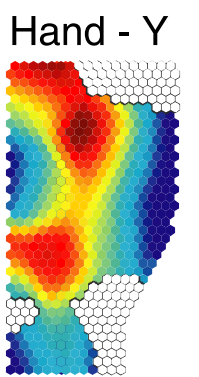

G

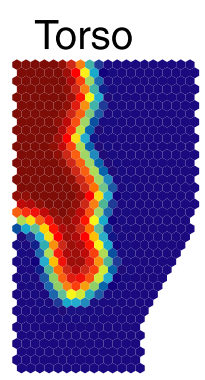

0

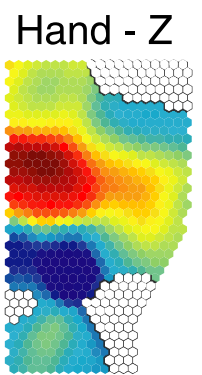

Figure 3. Final state of the self-organizing map model. In this learning set, the different movement types were presented to the network in equal proportion. $\boldsymbol{A}$, The initial somatotopic (Init. Somat.) arrangement of body parts before reorganization. $\boldsymbol{B}-\boldsymbol{K}$, Somatotopic arrangement of the 10 body parts after reorganization. $\boldsymbol{L}$, Arrangement of the five ethological categories after reorganization. In $\boldsymbol{L}$ only, light blue, hand-to-mouth; dark blue, reach; red, defense; green, central space/manipulation; pink, climbing. Regions of overlap have intermediate colors. $\boldsymbol{M}-\mathbf{0}$, Maps of hand location after reorganization. X, Hand height, with warm colors indicating greater height; $Y$, lateral location of hand, with warm colors indicating more lateral locations; Z, distance of hand from body along line of sight, with warm colors indicating more distant locations.

cal somatotopies such as a foot representation in a ventral position or a tongue representation in a dorsal position.

All of the methods for a Kohonen self-organizing network can be obtained from Kohonen (2001). In addition, a self-organizing network toolbox with standard programs can be obtained at http://www. cis.hut.fi/projects/somtoolbox/.

\section{Results}

Figure 3 shows the results from one learning set. Figure $3 A$ shows the initial somatotopic organization in the node array. For consistency with the spatial arrangement found in the monkey brain, in the following description, "posterior" refers to nodes on the right of the array, "anterior" to nodes on the left, "dorsal" and "medial" to nodes toward the top, and "ventral" and "lateral" to nodes toward the bottom of the array.

Figure $3 B-K$ shows the final somatotopic arrangement after learning. For example, $B$ shows the tongue representation, in which "hot" colors show the nodes that most strongly represent the tongue and "colder" colors show nodes that represent the tongue less strongly. These somatotopic panels show that the somatotopy became less discrete as a result of learning. The map became extensively overlapping.

The map contained more somatotopic overlap in more anterior areas and contained a more discrete somatotopy in the more posterior areas. Along the posterior edge of the map, a relatively discrete progression can be seen. This progression includes a mouth representation at the bottom, then a region that emphasizes the hand but also weakly represents the arm, then a region that emphasizes the arm but also weakly represents the hand, then a region that represents the foot and leg.

The hand representation became divided into three regions (Fig. $3 H$ ). One hand representation was located in the posterior part of the array, as if corresponding to the primary motor hand area; the second hand representation was located in an anterior region within the dorsal half of the array, as if corresponding to the dorsal premotor hand area; and the third hand representation was located in an anterior region at the ventral edge of the array, as if corresponding to the ventral premotor hand area (compare with Fig. $1 F$, based on results from the monkey brain.)

Figure $3 L$ shows the clustered arrangement of ethological movement categories after learning. Central-space/manipulation movements were represented in a posterior region, overlapping the posterior hand representation. Reaching movements were represented in an anterior dorsal region, overlapping the anterior dorsal hand representation. Hand-to-mouth movements were represented in an anterior ventral location, overlapping the anterior ventral hand representation. Defensive movements were represented in an anterior region between the hand-to-mouth representation and the reaching representation. Climbing movements were represented dorsal to the reaching movements, in the most dorsal and anterior corner of the map (compare with Fig. $1 I$, based on results from the monkey brain).

The particular pattern shown in Figure $3 L$, in which the different ethological categories are organized into a specific spatial arrangement, results from a bias provided by the initial somatotopic map. For example, the hand-to-mouth movements included the tongue, lips, jaw, neck, hand, and arm; the reaching movements included the arm, hand, and torso. Given the initial somatotopic locations for these body parts, the reaching movements inevitably came to be represented in a location dorsal to the handto-mouth movements. Similar initial somatotopic biases explain the relative location for the other ethological categories.

Figure $3 M-O$ shows the final topographic arrangement of hand position. In these maps, certain array locations are blanked out because the arm was not represented at these sites and therefore no hand location could be defined. The clearest organization was obtained for hand height. Upper hand positions were repre- 
A

Init. Somat.

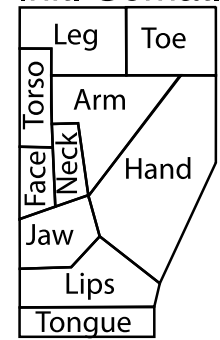

H

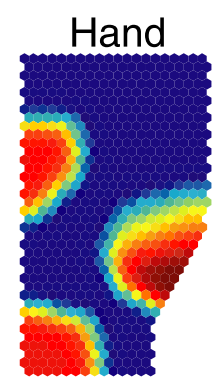

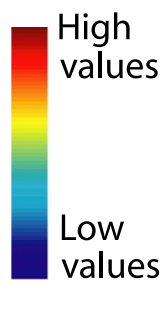

B

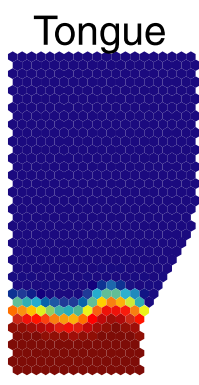

J

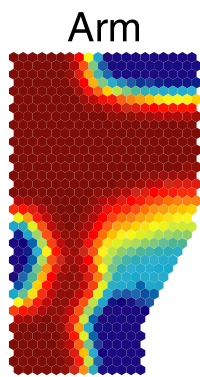

J

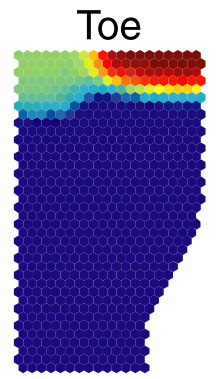

C

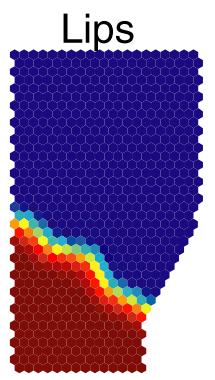

D

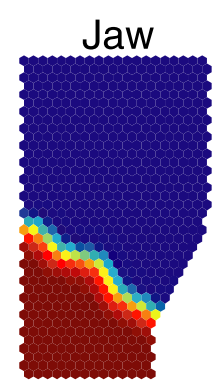

E

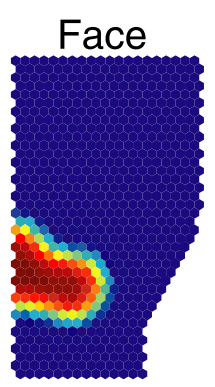

F

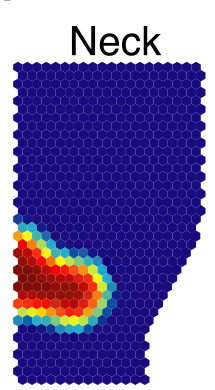

G

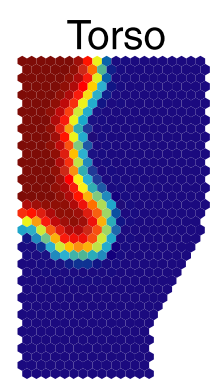

K

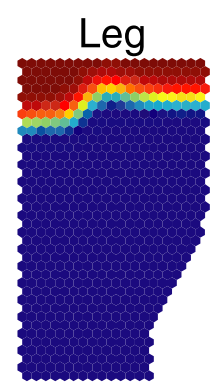

L

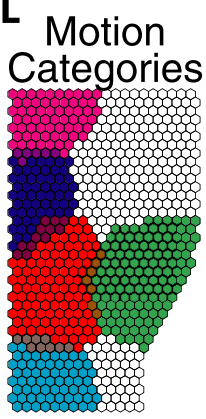

M

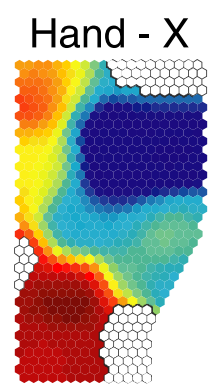

N

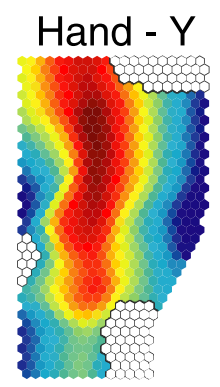

0

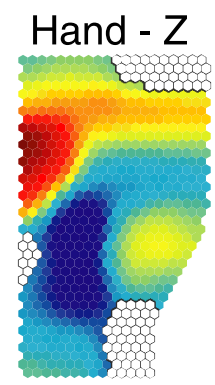

Figure 4. Alternate version of the self-organizing map model. In this learning set, different movement types were presented to the network in unequal proportions. The central-space/ manipulation movements were increased in proportion by $50 \%$, the reaching movements were decreased by $20 \%$, the hand-in-lower space movements were increased by $50 \%$, and the chewing movements and the leg and foot movements were decreased by $20 \%$. A similar result was obtained here as in Figure 3.

sented mainly in a ventral anterior area that included the handto-mouth zone; midheight locations were represented in an intermediate location that included the central-space/ manipulation zone; and lower hand locations were represented in a posterior dorsal area, completing the topographic progression. In addition to this map of hand height, there was also a second representation of upper hand locations in the anterior dorsal region of the array, corresponding to the climbing zone (compare with Fig. 1G, based on results from the monkey brain).

There was also some degree of mapping of the lateral-tomedial location of the hand, but with a reversal. Hand locations near the vertical meridian tended to be represented in posterior parts of the array, especially those corresponding to the centralspace/manipulation zone. Lateral hand locations tended to be represented in a more anterior strip of the array. In the reaching zone, in a dorsal anterior region, the map reversed and hand locations again tended to fall near the vertical meridian, representing hand locations distant from the body but near the midline (compare with Fig. $1 \mathrm{H}$, based on results from the monkey brain).

There was no clear systematic representation of the $z$ location of the hand (distance forward of the body along the line of sight), although in accordance with the tendency of a Kohonen map, the array showed a clumping in which similar $z$ values were represented in neighboring nodes.

\section{Alternate maps}

One possible concern with the result obtained in Figure 3 is that the model was trained on a movement set in which different movement categories were equally represented. The input array contained an equal proportion of all categories of movement. Monkeys do not perform these movements in equal proportions. For example, manipulation of objects in central space is a rela- tively high-frequency behavior, whereas reaching outward to grasp makes up a smaller proportion of the animal's spontaneous behavior (Graziano et al., 2003). Will the final configuration of the map depend on the exact proportions of these different movements in the training set? Figure 4 shows the results for a training set in which the different movement categories were represented in unequal proportions based on the observations of monkeys' spontaneous behavior in the home cage (for details, see figure legend). The results are nearly identical. The sizes of the different representations are slightly altered. For example, the region representing central-space/manipulation is slightly larger in Figure 4 than in Figure 3, reflecting the larger proportion of that category of movement in the learning set. The overall pattern of results, however, is similar.

To further probe the robustness of the final map, we tried other variants of the learning set and variants of the definitions of the movements. Figure 5 shows the arrangement of the ethological movement categories obtained from some of these variants (for details, see figure legend). The dimensionality reduction arrived at the same overall pattern. The final map is therefore highly constrained. If topographic continuity is to be optimized and if the map is to place importance on somatotopic organization, hand location, and ethological category, then the final arrangement that compromises among those different influences is essentially determined.

\section{Lesions and use-dependent changes}

Previous studies have shown that the topography in motor cortex can change with experience or with lesions to parts of motor cortex (Karni et al., 1995; Pascual-Leone et al., 1995; Nudo and Milliken, 1996; Nudo et al., 1996; Classen et al., 1998; Kleim et al., 1998). Can similar changes be observed in the present model of motor cortex? 


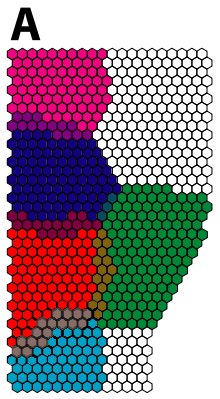

B

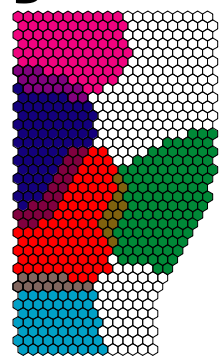

- Climbing/leaping

- Reach

- Hand to mouth

- Defensive

- Central Space/ Manipulation

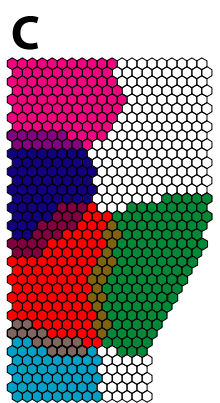

D

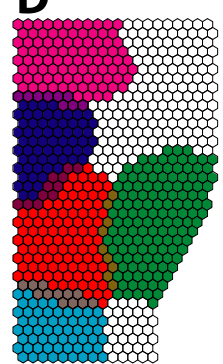

$\mathbf{F}$

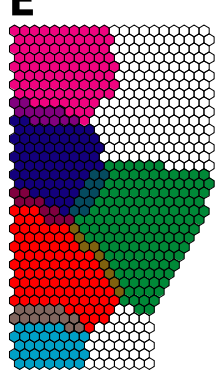

A

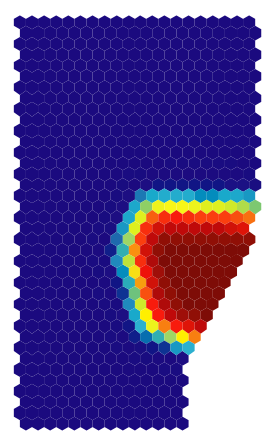

B

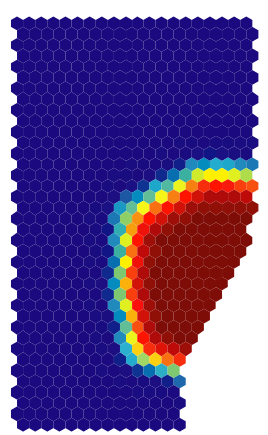

C

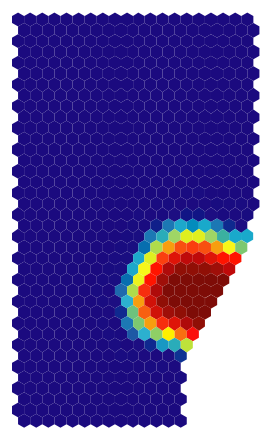

Figure 6. Usage-dependent changes in the map. The model was trained on the same movement set used in Figure 3 and then trained again on a second movement set. $\boldsymbol{A}$, Final state of map model when the second movement set was the same as the first. The representation of one ethological movement category, central-space/manipulation, is shown. $\boldsymbol{B}$, Final state of the map model when the second movement set was the same as the first except that the number of central-space/manipulation movements was increased by $50 \%$. C, Final state of the map model when the second movement set was the same as the first except that the number of centralspace/manipulation movements was decreased by $50 \%$.

representation. These blanked-out nodes were no longer functional parts of the array. Figure $7 A$ shows the hand representation and the lesion (black area) before any additional learning had occurred. Figure $7 B$ shows a similar plot for the arm representation. We then allowed the model to continue learning on the same standard movement set on which it had originally been trained. Figure $7 C$ shows the reorganized hand representation. Essentially, the residual hand representation around the edge of the lesion expanded to form a complete hand representation in an adjacent location. This reorganization of the hand representation also caused a reorganization in other parts of the map. This shifting of the rest of the map is illustrated in Figure $7 D$, in which the arm representation receded to allow space for the expansion of the hand representation.

One caveat is important to note here. The Kohonen network seeks an optimal map configuration. When map conditions are changed, such as when the usage of one type of movement is increased, or when a piece of the map is "lesioned," then the Kohonen algorithm seeks on a new optimal map configuration. In this sense, the present model shows use-dependent and lesiondependent changes. However, the process by which the Kohonen network finds the optimal map configuration is a purely computational one and is presumably different from the mechanisms of plasticity in the brain. The finding that the model settles on a similar final state as obtained in physiological studies of the brain suggests that the plastic mechanisms in the brain, whatever they may be, may also tend to seek continuity in mapping multiple parameters onto a two-dimensional sheet.

\section{Discussion}

The motor cortex map generated by our self-organizing network resembled the actual maps obtained from the lateral motor cortex of monkeys in five specific ways, listed below. the proportion of central-space/manipulation movements. The corresponding representation in the map expanded. Figure $6 \mathrm{C}$ shows the result when the second movement set included a 50\% decrease in the proportion of central-space/manipulation movements. The corresponding representation in the map shrank.

"Lesion" to the hand representation

We first let the model self-organize as in Figure 3. Once the model settled on a final map, we blanked out a set of nodes in the hand

\section{Simpler movements and clearer somatotopy in a posterior "primary-like" strip}

Traditionally, the posterior portion of motor cortex, "primary motor" cortex, is thought to contain a relatively more discrete somatotopy in which the major body parts are separated, whereas the more anterior strip, considered the "premotor" cortex, is thought to contain a more overlapping somatotopy and to con- 

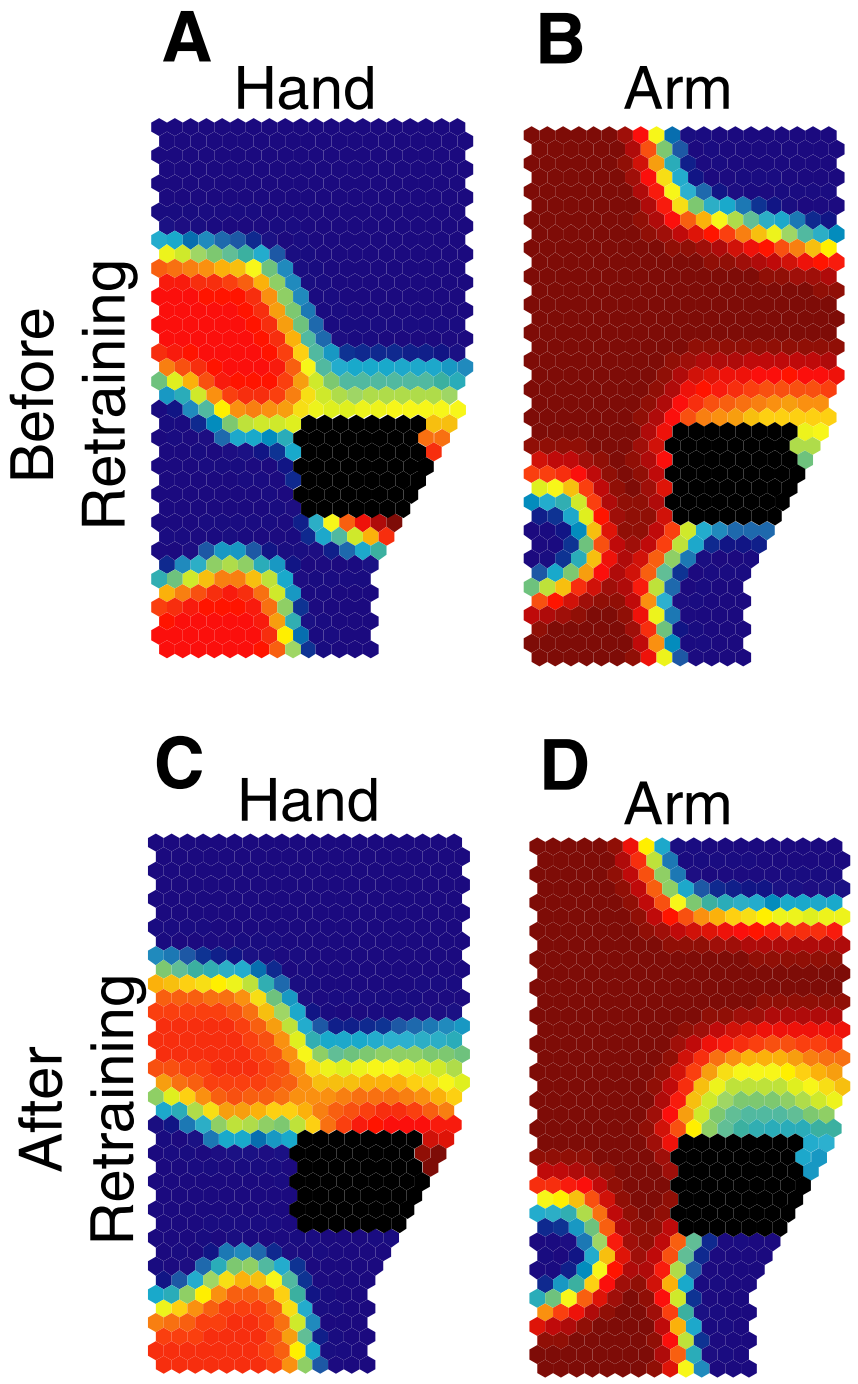

Figure 7. Effect of a "lesion" on the map model. $\boldsymbol{A}$, Final state of the hand representation using parameters and learning set as in Figure 3, with black area showing the site of the lesion to be made. $\boldsymbol{B}$, Same as $\boldsymbol{A}$ but for arm representation. $\boldsymbol{C}$, State of hand representation after lesion and additional learning on the same movement set. The lesioned hand representation expanded. $\boldsymbol{D}$, State of arm representation after lesion and additional learning on the same movement set. The arm representation shifted to allow room for the expanded hand representation.

trol more complex movements (Campbell, 1905; Fulton, 1938; Preuss et al., 1996; Rizzolatti and Luppino, 2001). Both strips project directly to the spinal cord (Bortoff and Strick, 1993; He et al., 1993; Dum and Strick, 1996, 2002; Wu et al., 2000), calling into question whether they form a true hierarchy or simply emphasize different types of movement.

The map generated by our self-organizing network contained similar topographic trends. In the posterior half of the array, there was a somatotopic sequence of four major representations, including the tongue, lips, and jaw (Fig. $4 B-D$ ), the hand (Fig. $3 H$ ), the arm (Fig. 3I), and the leg and foot (Fig. $3 J, K$ ). In the anterior regions, the overlap among body segments was more extensive and the somatotopy more fractured. The reason for this trend in the self-organizing map is clear: some of the movements in our model were especially complex in that they required coordination among different body parts. For example, reaching involved action of the hand, arm, and torso. Defensive movements involved action of the face, rotation of the head, movement of the torso, and movement of the arm. Climbing was perhaps the most integrated, involving the arm, torso, and leg. These movements involved the axial musculature, because the trunk and neck form the connecting links between different body segments. The initial somatotopy was arranged with the axial musculature in an anterior region and the distal musculature in a posterior region. As a result, during learning, the complex movements that link more than one body segment gravitated to the anterior regions of the map. Thus, in our model, in its final state, one can distinguish a posterior strip that is "primary-like" in that it contains a relatively discrete somatotopy, representing body segments in a separate manner, and an anterior strip that is "premotor-like" in that it contains a more integrated, blurred somatotopy and represents movements of greater intersegment complexity. However, no processing hierarchy is implied by the map. The anterior strip does not control the posterior strip.

\section{Cortical zones for ethologically relevant movement types}

Our previous stimulation experiments indicated that the precentral gyrus contains a mosaic of subregions or hot spots that emphasize different movement categories (Graziano et al., 2002, 2005; Cooke et al., 2004a,b). Stimulation in each subregion tended to evoke a specific type of movement recognizable from the monkey's behavioral repertoire. These five movement categories, obtained empirically on stimulation, were used to train our self-organizing map. During learning, the different movement types became clumped into distinct subregions of the array. Remarkably, the topographic arrangement of the subregions in the self-organizing map closely resembled the arrangement observed in the actual monkey brain. This arrangement of ethological zones is constrained by the initial somatotopy and the subsequent attempt of the network to optimize nearest-neighbor relationships.

\section{Three hand representations}

Three main hand representations have been described in the lateral precentral gyrus of monkeys: one in a posterior area in primary motor cortex, one in dorsal premotor cortex (probably in the caudal division), and one in ventral premotor cortex. The three hand areas were defined by their projections to the hand motoneuron pools in the spinal cord (He et al., 1993; Dum and Strick, 2002, 2005). Our self-organizing map also converged on three hand representations (Fig. $4 H$ ). The reason why the network arrived at three hand representations is that the hand participated in three distinct movement categories. One hand representation was on the posterior edge of the array, emphasizing the distal movements used during manipulation of objects. The second hand area was in the dorsal anterior portion of the array, emphasizing the control of reaching to grasp. The third hand area was in the ventral anterior portion of the array, emphasizing interactions between the hand and the mouth.

\section{Noisy maps of hand location}

We previously found that stimulation of different sites in the monkey motor cortex drove the hand to different locations in space and that the evoked hand locations formed a map across the lateral motor strip, encompassing the primary motor and caudal premotor areas (Graziano et al., 2002, 2003, 2005). The map contained considerable overlap. The height of the hand was most consistently mapped, the lateral position of the hand was less clearly ordered in the map, and the forward distance of the hand along the line of sight showed no consistent topography. Our self-organizing map converged on a topographic arrangement of 
hand position that roughly resembled this arrangement seen in monkeys.

\section{Learning-dependent reorganization}

Our self-organizing network began with a discrete, well defined somatotopy and then became reorganized during training on a set of complex movements. Data from motor cortex in humans, monkeys, cats, and rats suggest that a similar process occurs during development and learning, in which an initial somatotopy reorganizes around the types of movement that are common in the animal's repertoire. In the cat, at an early stage in development, the motor cortex contains a discrete somatotopic map in which joints have separate representation, but, during practice with more complex reaching movements, the map develops overlapping representations of functionally related joints (Martin et al., 2005). In humans, rats, and monkeys, practice on a motor task can cause changes in the size and degree of overlap of the cortical representations of the relevant body parts (Karni et al., 1995; Pascual-Leone et al., 1995; Nudo et al., 1996; Classen et al., 1998; Kleim et al., 1998). In humans, practice on a task that combines movements of more than one body part can cause the representations of those body parts to move toward each other (Liepert et al., 1999). These and other studies suggest that motor cortex topography is plastic and constantly reorganizing, and movement components that normally occur together during behavior can develop representations that are near each other or overlapping in cortex. This topographic reorganization has been suggested to be a physical manifestation of the "motor engram," in which memory for complex motor skills is stored (Monfils et al., 2005). We suggest that it is precisely these motor engrams that are triggered by electrical stimulation. Complex, behaviorally relevant movements, electrically evoked from specific sites in motorrelated cortical areas, have now been observed in monkeys, rats, cats, and prosimians (Ethier et al., 2004; Haiss and Schwarz, 2005; Stepniewska et al., 2005).

\section{Differences between the model and the monkey data}

In the monkey, the hand representation is reported to be surrounded in a semicircle by a forearm and upper arm representation (Fig. 1C) (Kwan et al., 1978; Park et al., 2001). In our model, the arm representation also tended to wrap partially around the hand representation (Fig. 4H,I). This wrap-around was less complete in our model than in the descriptions from the monkey. One possible reason is that the model contained only a crude somatotopy including a hand and an arm, but not the finer gradations of wrist, forearm, elbow, and shoulder. Thus, the wrapping of the wrist and forearm representation around the hand was not possible here.

A second difference between the model and the data are that the model resulted in nearly identical maps on different runs, despite changes in the proportions of different movements, yet the maps in the monkey appear to be variable among individuals. Some possible reasons for the greater variation in monkeys are as follows: the exact size and shape of the relevant motor strip may be different in different individuals, leading to different proportions internal to the strip; and the actual motor map may be influenced by many more factors than the three modeled here.

Despite these differences, by using only three main mapping factors in competition with each other, the model was able to produce a topography that captures some of the essential features of the monkey motor cortex. We suggest that the organization in motor cortex cannot be understood as a single map of one kind or another, but rather can be partially understood as a result of an initial, underlying somatotopy that is reorganized under the competing influences of other mapping requisites, including a cluster map of ethologically relevant movements and a topographic map of hand position in space. Other variables relevant to the animal's movement repertoire may also influence the topography within motor cortex in ways not tested in the present model.

\section{References}

Bortoff GA, Strick PL (1993) Corticospinal terminations in two new-world primates: further evidence that corticomotoneuronal connections provide part of the neural substrate for manual dexterity. J Neurosci 13:5105-5118.

Campbell AW (1905) Histological studies on the localization of cerebral function. New York: Cambridge UP.

Classen J, Liepert J, Wise SP, Hallett M, Cohen LG (1998) Rapid plasticity of human cortical movement representation induced by practice. J Neurophysiol 79:1117-1123.

Cooke DF, Graziano MSA (2003) Defensive movements evoked by air puff in monkeys. J Neurophysiol 90:3317-3329.

Cooke DF, Graziano MSA (2004a) Sensorimotor integration in the precentral gyrus: polysensory neurons and defensive movements. J Neurophysiol 91:1648-1660.

Cooke DF, Graziano MSA (2004b) Super-flinchers and nerves of steel: defensive movements altered by chemical manipulation of a cortical motor area. Neuron 43:585-593.

Dum RP, Strick PL (1996) Spinal cord terminations of the medial wall motor areas in macaque monkeys. J Neurosci 16:6513-6525.

Dum RP, Strick PL (2002) Motor areas in the frontal lobe of the primate. Physiol Behav 77:677-682.

Dum RP, Strick PL (2005) Frontal lobe inputs to the digit representations of the motor areas on the lateral surface of the hemisphere. J Neurosci 25:1375-1386.

Durbin R, Mitchison G (1990) A dimension reduction framework for understanding cortical maps. Nature 343:644-647.

Ethier C, Imbeault M, Ung VR, Capaday C (2004) Vectorial addition of motor cortical outputs in the cat. Soc Neursci Abstr 30:872.14.

Foerster O (1936) The motor cortex of man in the light of Hughlings Jackson's doctrines. Brain 59:135-159.

Fritsch G, Hitzig E (1870) Ueber die elektrishe Erregarkeit des Grosshirns (von Bonin G, translator). In: The cerebral cortex (Nowinski WW, ed), pp 73-96. Springfield, IL: Thomas.

Fulton JF (1938) Physiology of the nervous system, pp 399-457. New York: Oxford UP.

Graziano MSA, Taylor CSR, Moore T (2002) Complex movements evoked by microstimulation of precentral cortex. Neuron 34:841-851.

Graziano MSA, Cooke DF, Taylor CSR, Moore T (2003) Distribution of hand location in monkeys during spontaneous behavior. Exp Brain Res 155:30-36.

Graziano MSA, Patel KT, Taylor CSR (2004) Mapping from motor cortex to biceps and triceps altered by elbow angle. J Neurophysiol 92:395-407.

Graziano MSA, Aflalo T, Cooke DF (2005) Arm movements evoked by electrical stimulation in the motor cortex of monkeys. J Neurophysiol 94:4209-4223.

Haiss F, Schwarz C (2005) Spatial segregation of different modes of movement control in the whisker representation of rat primary motor cortex. J Neurosci 25:1579-1587.

He SQ, Dum RP, Strick PL (1993) Topographic organization of corticospinal projections from the frontal lobe: motor areas on the lateral surface of the hemisphere. J Neurosci 13:952-980.

Karni A, Meyer G, Jezzard P, Adams MM, Turner R, Ungerleider LG (1995) Functional MRI evidence for adult motor cortex plasticity during motor skill learning. Nature 377:155-158.

Kleim JA, Barbay S, Nudo RJ (1998) Functional reorganization of the rat motor cortex following motor skill learning. J Neurophysiol 80:3321-3325.

Kohonen T (1984) Self organizing maps and associative memory. Berlin: Springer.

Kohonen T (2001) Self-organizing maps. Berlin: Springer.

Kwan HC, MacKay WA, Murphy JT, Wong YC (1978) Spatial organization of precentral cortex in awake primates. II. Motor outputs. J Neurophysiol 41:1120-1131. 
Liepert J, Terborg C, Weiller C (1999) Motor plasticity induced by synchronized thumb and foot movements. Exp Brain Res 125:435-439.

Martin JH, Engber D, Meng Z (2005) Effect of forelimb use on postnatal development of the forelimb motor representation in primary motor cortex of the cat. J Neurophysiol 93:2822-2831.

Matelli M, Luppino G, Rizzolatti G (1985) Patterns of cytochrome oxidase activity in the frontal agranular cortex of the macaque monkey. Behav Brain Res 18:125-136.

Monfils MH, Plautz EJ, Kleim JA (2005) In search of the motor engram: motor map plasticity as a mechanism for encoding motor experience. The Neuroscientist 11:471-483.

Nudo RJ, Milliken GW (1996) Reorganization of movement representations in primary motor cortex following focal ischemic infarcts in adult squirrel monkeys. J Neurophysiol 75:2144-2149.

Nudo RJ, Milliken GW, Jenkins WM, Merzenich MM (1996) Usedependent alterations of movement representations in primary motor cortex of adult squirrel monkeys. J Neurosci 16:785-807.

Obermayer K, Blasdel GG (1993) Geometry of orientation and ocular dominance columns in monkey striate cortex. J Neurosci 13:4114-4129.

Pascual-Leone A, Nguyet D, Cohen LG, Brasil-Neto JP, Cammarota A, Hallett M (1995) Modulation of muscle responses evoked by transcranial magnetic stimulation during the acquisition of new fine motor skills. J Neurophysiol 74:1037-1045.
Park MC, Belhaj-Saif A, Gordon M, Cheney PD (2001) Consistent features in the forelimb representation of primary motor cortex in rhesus macaques. J Neurosci 21:2784-2792.

Preuss TM, Stepniewska I, Kaas JH (1996) Movement representation in the dorsal and ventral premotor areas of owl monkeys: a microstimulation study. J Comp Neurol 371:649-676.

Rizzolatti G, Luppino G (2001) The cortical motor system. Neuron 31:889-901.

Schreiner CE (1995) Order and disorder in auditory cortical maps. Curr Opin Neurobiol 5:489-496.

Stepniewska I, Fang PC, Kaas JH (2005) Microstimulation reveals specialized subregions for different complex movements in posterior parietal cortex of prosimian galagos. Proc Natl Acad Sci USA 102:4878-4883.

Woolsey CN, Settlage PH, Meyer DR, Sencer W, Hamuy TP, Travis AM (1952) Pattern of localization in precentral and "supplementary" motor areas and their relation to the concept of a premotor area. In: Association for research in nervous and mental disease, Vol 30, pp 238-264. New York: Raven.

Wu CW, Bichot NP, Kaas JH (2000) Converging evidence from microstimulation, architecture, and connections for multiple motor areas in the frontal and cingulate cortex of prosimian primates. J Comp Neurol 423: $140-177$. 